\title{
QUANTUM CONCENTRATION FOR THE LIQUID PHASE OF ELEMENTS
}

\author{
Askar Kassymov ${ }^{1,2}$, Hanno Schaumburg ${ }^{2}$, Serik E. Kumekov ${ }^{3}$, Karen S. Martirosyan ${ }^{1 *}$ \\ ${ }^{1}$ University of Texas at Brownsville, \\ Department of Physics and Astronomy, Brownsville, Texas, 78520, USA \\ ${ }^{2}$ Hamburg University of Technology, 21073 Hamburg, Germany \\ ${ }^{3}$ Kazakh National Technical University after K.I. Satpaev, 050013 Almaty, Kazakhstan \\ *-Email: karen.martirosyan@utb.edu
}

The novel concept of quantum concentration for the liquid phase of elements was introduced which indicates the number of possible states of atoms per unit volume. A simplified thermodynamic energy model for a solid-liquid phase transition is used to define the quantum concentration for the liquids. Values of quantum concentration for liquid phase of most of elements were calculated and analyzed. It was revealed that the metalloids such as boron, silicon, germanium, antimony, tellurium and bismuth exhibited a highest value of quantum concentrations compared with other elements. Several physical and chemical properties of substances (such as melting temperature and free energy) can be calculated using these data. The size dependence of melting temperatures of $\mathrm{Au}, \mathrm{Pb}$ and $\mathrm{Sn}$ spherically symmetric nanoparticles were calculated and compared well with experimental data and theoretical model based on generalized Gibbs-Thomson predictions.

Keywords: elements, nanostructures, melting temperature, interfaces, phase equilibria 


\section{Introduction}

For the past century quantum theory has been an essential part of the modern physics and quantized energy levels, spin, the geometry of electronic orbitals, and the Pauli Principle can all be applied to understand the behavior of the single atom, extending to atom clusters, small molecules, and finally to the properties of matter [1]. This provides a substantial useful framework for various features of the modern elements including the behavior of atoms during chemical bonding and has played a significant role in the development of numerous modern technologies. For example, the application of the quantum concentration concept in an ideal gas as pioneered by Kittel and Kroemer [2] has made quantum states "countable" and therefore accessible for a statistical calculation of entropies. The quantum concentration (number of states per volume) of an ideal gas is determined by the following equation:

$$
\rho_{Q}=\left(\frac{2 \pi M k T}{h^{2}}\right)^{3 / 2}
$$

where $M$ is the particle mass, $T$ is the absolute temperature, $k$ is the Boltzmann constant and $h$ is Planck constant. Since the quantum concentration of the ideal gas is limited, it would be contradictory if the concentration of available states in a liquid would be infinite, therefore it should be countable as well and can be treated statistically. Let consider a system with one kind of particles with the particle number $n$ that can be distributed over available sites with the number $N$. The number of particles per one available site is $c=n / N$.

The identical average particle energy $W_{n}$ and the entropy of distribution (mixing over available states) $S^{M}$ the free energy can be expressed by using Helmholtz free energy (F) approximation:

$$
F=n \cdot W_{n}-T \cdot S^{M}
$$


Using typical permutation statistics as described in several textbooks on thermodynamics and materials science [1-4] and solid state electronics [5-8] using Eq. (2), we define the entropy of distribution per particle the following equations:

$$
\begin{aligned}
& S=S^{M}(N, n)=+k \cdot\{N \ln N-(N-n) \ln (N-n)\} \\
& \Rightarrow S^{M}(N, c)=-k \cdot N \cdot\{c \ln c+(1-c) \ln (1-c)\}
\end{aligned}
$$

This formalism can be effectively used in materials science as well as in condensed matter physics. While in standard theory the entropy of distribution is used for the mixing of $n$ impurity atoms in a matrix of $N$ atoms we consider here the distribution of $n$ atoms of one kind over $N_{Q}$ quantum states that the particles can occupy:

$$
c=\frac{n}{N_{Q}}
$$

Here $c$ means the number of $n$ atoms per $N_{Q}$ quantum states.

In this work we introduce a novel concept for a statistical interpretation of the entropy of melting using the quantum concentration for the liquid phase $n_{Q L}$, which allows for a statistical calculation of the free energy in the liquid. The quantum concentration of liquids can be determined using a geometrical relation derived from the thermodynamic energy model for a solid-liquid phase transition. The thermodynamic energy model to calculate the size dependence of the melting temperature of spherically symmetric nanoparticles is considered. The relation between the results and limited experimental observations is also discussed.

\section{Thermodynamic energy model for a solid-liquid phase transition}

The diagram of the thermodynamic energy model for a solid-liquid phase transition is a simplification of the standard thermodynamic model for alloys [1-4] that is valid for systems without phase separation presented in Figure 1. According to the considered model 
the free energy per atom for solid and liquid phases are expressed through the following relations:

$$
\begin{aligned}
& F^{\text {liqu }}\left(n, n_{Q}\right)=n \cdot\left(W_{s}+W_{\text {lat.heat }}\right)+k T \cdot n_{Q} \cdot\left\{c_{l} \ln c_{l}+\left(1-c_{l}\right) \ln \left(1-c_{l}\right)\right\} \\
& \Rightarrow f^{\text {liqu }}\left(n, n_{Q}\right):=\frac{F^{\text {liqu }}}{n}=W_{s}+W_{\text {lat.heat }}+\frac{k T}{c_{l}}\left\{c_{l} \ln c_{l}+\left(1-c_{l}\right) \ln \left(1-c_{l}\right)\right\}
\end{aligned}
$$

Where $f^{\text {solid }}:=\frac{F^{\text {solid }}}{n}=W_{s} \quad W_{\text {lat.heat }}$ is latent heat of fusion per atom, $W_{s}$ is energy per atom in the solid phase, and $c_{l}=\frac{n}{n_{Q L}}$ is the number of atoms of the pure phase divided by the number of available states in the liquid phase with the physical meaning of an occupation rate.

At the melting point $T_{m}$ the free energies in solid and liquid phases are equal to each other:

$$
f^{\text {solid }}=f^{\text {liquid }}
$$

thus from eq. (6) it follows:

$$
W_{\text {lat.heat }}=-\frac{k T_{m}}{c_{l}}\left\{c_{l} \ln c_{l}+\left(1-c_{l}\right) \ln \left(1-c_{l}\right)\right\}
$$

The thermodynamic energy model for a solid-liquid phase transition has an important geometrical interpretation, since the curve representing the free energy per atom of the liquid phase is fixed at the temperature $T=0$ (it has the value of $W_{s}$ plus $W_{\text {lat.heat }}$ ) and at the melting temperature $T_{m}$. According to equation (3) the slope is corresponding to the negative entropy per atom $S^{M} / n$. Decreasing entropy per atom lead to increase $c_{l}$ that expends a fewer additional occupational states in the liquid phase as compared to the solid phase.

\section{Quantum concentration of the liquid}

Since the quantum concentration of an ideal gas is limited, the quantum concentration of the liquid similarly has to be limited and most likely should be lower. It can directly determined from equation (8) the following relation:

$$
\frac{W_{\text {lat.heat }}}{k T_{m}}=-\frac{1}{c_{l}}\left\{c_{l} \ln c_{l}+\left(1-c_{l}\right) \ln \left(1-c_{l}\right)\right\}
$$


The quantum concentration of the liquid phase of elements is presented in Figure 2 as a tabular arrangement of the chemical elements in the periodic table form. Experimental data such as melting temperature, density of liquid phase and latent heat of fusion were taken from CHEMIX 3.5 database and Ref. [9]. The comparison of the quantum concentration of the liquid phase of elements with concentration of atoms is presented in Figure 3. As it is shown in the Figures 2 and 3 metalloids elements such as boron, silicon, germanium, antimony, tellurium and bismuth exhibited highest value of quantum concentrations for the liquid phase. From equation (9) it follows that this is due to a relatively high value of $W_{\text {lat.heat }} / k T_{m}$ that for many elements (with a hexagonal and cubic structure with coordination number $\geq 8$ [3]) is the order of 1 . There are not available experimental data for the latent heat of fusion for some elements (He, C, As, Pm, Po, At, Fr, Ra, Ac, Cm, Bk etc), thus, quantum concentrations for these elements cannot be calculated.

\section{Size dependence of the melting temperature}

In the last decade, there has been incredible progress in condensed matter physics at the nanostructured level and the development of several new concepts has provided a variety perspective on the size dependent phenomena in condensed matter systems. In the framework of classical thermodynamics, melting of a solid is defined as a first-order discontinuous phase transformation following at a critical temperature at which Gibbs free energies of the solid and the liquid states are equal. Numerous experimental observations verified that melting temperature of nanoparticles decreases with decreasing of the particle size [10-18]. Melting point depression is a very important issue for materials and device applications containing nanostructured particles, as it decreases the functional range of the solid phase and increases the chemical reactivity [19].

For the calculation of the melting point depression we consider a sphere of a solid phase with diameter $d$ existing in a liquid environment of the same element. The surface 
atoms on the solid-liquid interface share approximately half of their binding energy between solid and liquid phases $[3,15]$, thus raising their energy as follow:

$$
\Delta W_{\text {surf }} \approx \frac{1}{2} W_{\text {lat.heat }}
$$

Then the total surface energy per atom in the growing solid is

$$
\Delta W_{\text {nsurf }}=\frac{n_{\text {surf }} \Delta W_{\text {surf }}}{n_{\text {vol }}}=6 \frac{d_{\text {atom }}}{d} \Delta W_{\text {surf }}
$$

where $d_{\text {atom }}$ is atomic diameter and $d$ is diameter of the nanoparticle.

In the thermodynamic energy model for a solid-liquid phase transition for nanoparticles presented in Figure 4, $\Delta W_{\text {nsurf }}$ has to be added to the free energy per atom of the solid phase:

$$
f_{\text {solid }}^{\text {nano }}=W_{s}+\Delta W_{\text {nsurf }}
$$

Equation for free energy per atom of liquid remains the same as in equation (6). Comparing the expressions for the free energy per atom of solid and liquid phases we can clearly calculate the melting temperature of nanoparticles:

$$
\begin{aligned}
& f_{\text {nano }}^{\text {solid }}=f^{\text {liquid }} \\
& \Rightarrow T_{m}=\frac{W_{\text {nsurf }}-W_{\text {lat.heat }}}{\frac{k}{c_{l}}\left\{c_{l} \ln c_{l}+\left(1-c_{l}\right) \ln \left(1-c_{l}\right)\right\}}
\end{aligned}
$$

Inserting (8) and (11) leads to this relation

$$
\frac{T_{m}^{\text {nano }}}{T_{m}}=1-3 \cdot \frac{d_{\text {atom }}}{d}
$$

This relation also derived in [12] from different assumptions that exhibit a basic property of nanoparticles. Eq (14) also could be directly geometrically determined from the thermodynamic energy model presented in Figure 4 that demonstrated the diagram of the thermodynamic model for a solid-liquid phase transition for nanoparticles.

Figure 5 shows comparison of the experimentally measured melting temperature versus particle diameter for $\mathrm{Au}, \mathrm{Pb}, \mathrm{Sn}$ and our theoretical predictions. The melting 
temperature of a nanoparticle decreases sharply as the particle reaches critical diameter in the range of $10-50 \mathrm{~nm}$. This behavior explained from a simple thermodynamic argument using the surface energy and the surface-to-volume ratio. Thus, the calculated size dependence of melting temperatures of $\mathrm{Au}, \mathrm{Pb}$ and $\mathrm{Sn}$ nanoparticles compared well with limited experimental data and the model [14] based on generalized Gibbs-Thomson theoretical predictions. The proposed model can easily be modified and applied to understand the melting behavior of various nanoparticles. Overall, thermodynamic model can be used to understand the size-dependent melting of nanoparticles for the intermetallic and other composite materials.

\section{Conclusions}

A novel concept of the quantum concentration for the liquid was introduced by using thermodynamic energy model for a solid-liquid phase transition. The calculation of quantum concentration for the liquid of metalloids such as boron, silicon, germanium, antimony, tellurium and bismuth demonstrated a highest value of compared with other elements. The mathematical model for the estimation of melting temperature of a spherical nanoparticles was established by using quantum concentration for the liquids. Size dependence of melting temperatures of $\mathrm{Au}, \mathrm{Pb}$ and $\mathrm{Sn}$ nanoparticles were estimated and compared well with experimental data and theoretical predictions. The quantum concentration concept well suited for a statistical calculation of the thermodynamic properties of liquids and therefore quite valuable for the calculation of phase diagrams using Gibbs thermodynamics. The quantum concentration for the liquid phase of elements can potentially create an interactive tool for the exploration and visualization of quantum behavior of matter and it can be extended to correlate melting temperature upon the arrangement of the atoms in the crystal lattice as well as the strength of the pairwise group interactions. 


\section{Acknowledgments}

We acknowledge the financial support of this research by the National Science Foundation (Grants 1138205 and HRD-1242090) and Committee of Science at Ministry of Education and Science Republic of Kazakhstan grant № 1037/ГФ4. 


\section{References}

1. Ch. Kittel and H. Krömer, Thermal Physics (W. H. Freeman and Company, San Francisco, 2000), p. 473.

2. Ch. Kittel and H. Krömer, Thermodynamik (Oldenburg Verlag, München, 2013), p. 463.

3. P. Haasen, Physikalische Metallkunde (Springer Verlag, Berlin-Heidelberg, 1994), p. 368.

4. H. Schaumburg, Werkstoffe (B. G. Teubner Verlag, Stuttgart, 1990), p. 398.

5. H. Schaumburg, Halbleiter (B. G. Teubner Verlag, Stuttgart, 1991), p. 614.

6. H. Schaumburg, Sensoren (B. G. Teubner Verlag, Stuttgart, 1992), p. 517.

7. Ch. Kittel, Introduction to Solid State Physics (John Wiley and Sons, New York, 2005), p. 704.

8. Ch. Kittel, Einführung in die Festkörperphysik (Oldenburg Verlag, München, 2013), p. 754.

9. CRC Handbook of Chemistry and Physics (CRC Press, Boca Raton, 2005), p. 2660.

10. Q. Jiang, S. Zhang, M. Zhao, Materials Chemistry and Physics, 82, 225-227 (2003).

11. L.H. Liang, J.C. Li, Q. Jiang, Physica B 334, 49-53 (2003).

12. W.H. Qi, M.P. Wang, Materials Chemistry and Physics, 88, 280-284 (2004).

13. K.K. Nanda, Chem. Phys. Lett. 419, 195-200 (2006).

14. W.H. Qi, Physica B, 368, 46-50 (2005).

15. A.H. Cottrell, Theoretical Structural Metallurgy (Edward Arnold Ltd, London, 1965), p. 251.

16. T.B. David, Y. Lereah, G. Deutscher, et al., Philos. Mag. A 71, 1135 (1995).

17. S.L. Lai, J.Y. Guo, V. Petrova, et al., Phys. Rev. Lett. 77, 99 (1996).

18. G. Schmid, B. Corain, Eur. J. Inorg. Chem. 17, 3081-3098 (2003).

19. K.S. Martirosyan, J. Mater. Chem., 21, 9400-9405, (2011). 


\section{FIGURES}

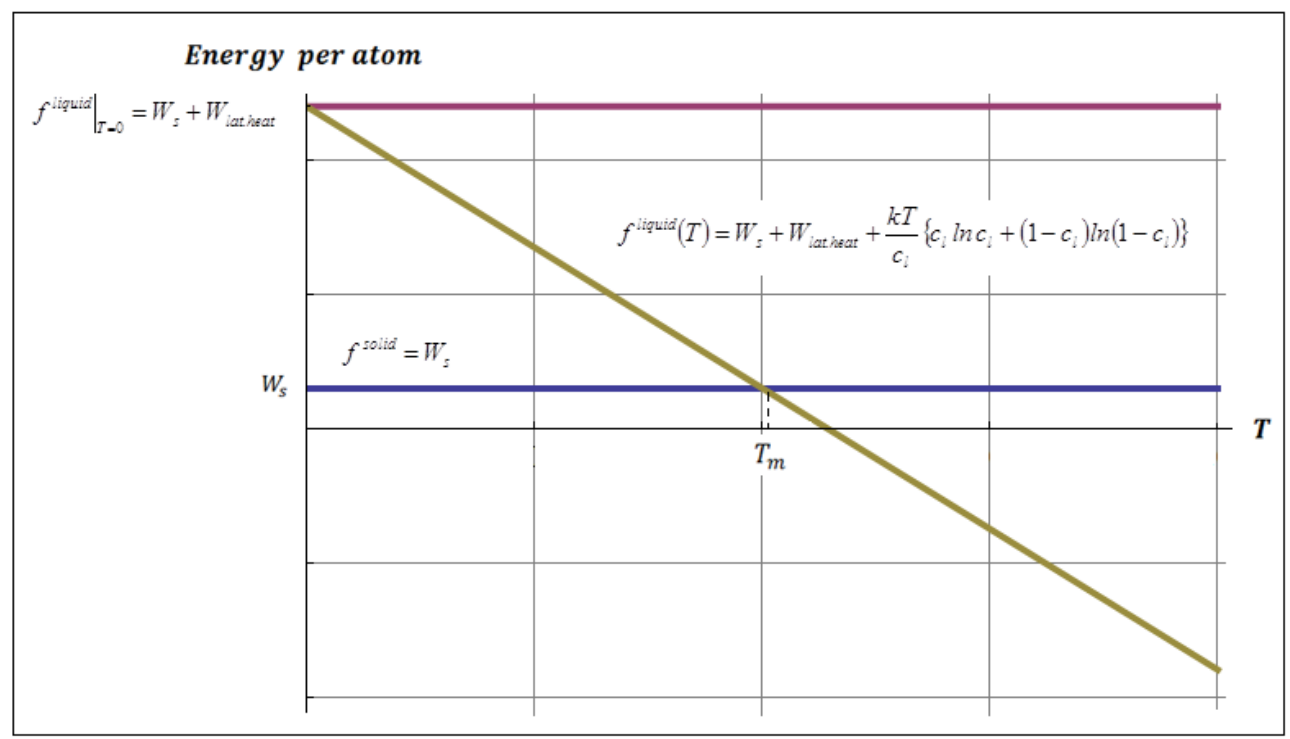

Figure 1. Diagram of the thermodynamic energy model for a solid-liquid phase transition. 


\section{Quantum concentration for the liquid phase of elements}

\begin{tabular}{|c|c|c|c|c|c|c|c|c|c|c|c|c|c|c|c|c|c|}
\hline \multirow{2}{*}{\multicolumn{17}{|c|}{ (2) }} & \multirow{3}{*}{$\begin{array}{c}2 \mathrm{He} \\
\mathrm{n} / \mathrm{a} \\
10 \mathrm{Ne}\end{array}$} \\
\hline & & & & & & & & & & & & & & & & & \\
\hline $3 \mathrm{Li}$ & $4 \mathrm{Be}$ & & & & & & & & & & & $5 \mathrm{~B}$ & $6 \mathrm{C}$ & $7 \mathrm{~N}$ & 80 & $9 \mathrm{~F}$ & \\
\hline 0,99 & 2,91 & & & & & & & & & & & 12,15 & $\mathrm{n} / \mathrm{a}$ & 0,69 & 0,7 & 0,85 & 1,83 \\
\hline $11 \mathrm{Na}$ & $12 \mathrm{Mg}$ & & & & & & & & & & & $13 \mathrm{Al}$ & $14 \mathrm{Si}$ & $15 \mathrm{P}$ & $16 \mathrm{~s}$ & $17 \mathrm{Cl}$ & $18 \mathrm{Ar}$ \\
\hline 0,56 & 1,26 & & & & & & & & & & & 2,13 & 20,52 & 0,45 & 0,58 & 2,49 & 1,16 \\
\hline $19 \mathrm{~K}$ & $20 \mathrm{Ca}$ & $21 \mathrm{Sc}$ & $22 \mathrm{Ti}$ & $23 \mathrm{~V}$ & $24 \mathrm{Cr}$ & $25 \mathrm{Mn}$ & $26 \mathrm{Fe}$ & $27 \mathrm{Co}$ & $28 \mathrm{Ni}$ & $29 \mathrm{Cu}$ & $30 \mathrm{Zn}$ & $31 \mathrm{Ga}$ & $32 \mathrm{Ge}$ & $33 \mathrm{As}$ & $34 \mathrm{Se}$ & $35 \mathrm{Br}$ & $36 \mathrm{Kr}$ \\
\hline $\begin{array}{r}0,29 \\
\end{array}$ & $\begin{array}{r}0,52 \\
\end{array}$ & 0,96 & 1,35 & 2,08 & 1,9 & 1,7 & 1,89 & 2,39 & 2,71 & 2,42 & 2,16 & $\begin{array}{r}4,85 \\
\end{array}$ & 18,33 & $n / a$ & 1,58 & 2,58 & 1,02 \\
\hline $37 \mathrm{Rb}$ & $38 \mathrm{Sr}$ & $39 \mathrm{Y}$ & $40 \mathrm{Zr}$ & $41 \mathrm{Nb}$ & $42 \mathrm{Mo}$ & $43 \mathrm{Tc}$ & $44 \mathrm{Ru}$ & $45 \mathrm{Rh}$ & $46 \mathrm{Pd}$ & $47 \mathrm{Ag}$ & $48 \mathrm{~cd}$ & $49 \ln$ & $50 \mathrm{Sn}$ & $51 \mathrm{Sb}$ & $52 \mathrm{Te}$ & 531 & $54 \mathrm{Xe}$ \\
\hline 0,24 & 1,25 & 0,62 & 1 & 1,74 & 2,22 & 2,3 & 1,94 & 1,99 & 1,87 & 1,57 & 1,5 & 0,92 & 1,9 & 4,56 & 4,96 & 2,68 & 0,78 \\
\hline $55 \mathrm{Cs}$ & $56 \mathrm{Ba}$ & $57-71$ & $72 \mathrm{Hf}$ & $73 \mathrm{Ta}$ & $74 \mathrm{~W}$ & $75 \mathrm{Re}$ & 76 Os & $77 \mathrm{Ir}$ & $78 \mathrm{Pt}$ & $79 \mathrm{Au}$ & $80 \mathrm{Hg}$ & $81 \mathrm{Tl}$ & $82 \mathrm{~Pb}$ & $83 \mathrm{Bi}$ & 84 Po & 85 At & $86 \mathrm{Rn}$ \\
\hline 0,19 & 0,37 & Lanthanides & 1,29 & 1,6 & 1,83 & 1,95 & 2,01 & 1,92 & 1,94 & 1,64 & 1,33 & 0,79 & 0,81 & 3,53 & $n / a$ & $n / a$ & $n / a$ \\
\hline $87 \mathrm{Fr}$ & $88 \mathrm{Ra}$ & $89-103$ & $104 \mathrm{Rf}$ & $105 \mathrm{Db}$ & $106 \mathrm{Sg}$ & $107 \mathrm{Bh}$ & $108 \mathrm{Hs}$ & $109 \mathrm{Mt}$ & 110 Ds & $111 \mathrm{Rg}$ & $112 \mathrm{Cn}$ & 113 Uut & $114 \mathrm{FI}$ & 115 Uup & $116 \mathrm{Lv}$ & 117 Uus & 118 Uno \\
\hline$n / a$ & $n / a$ & Actinides & $n / a$ & $n / a$ & $n / a$ & $\mathrm{n} / \mathrm{a}$ & $n / a$ & $n / a$ & $n / a$ & $n / a$ & $n / a$ & $n / a$ & $n / a$ & $n / a$ & $n / a$ & $n / a$ & $n / a$ \\
\hline & & Lanthanides & 57 La & $58 \mathrm{Ce}$ & $59 \mathrm{Pr}$ & $60 \mathrm{Nd}$ & $61 \mathrm{Pm}$ & $62 \mathrm{Sm}$ & $63 \mathrm{Eu}$ & $64 \mathrm{Gd}$ & $65 \mathrm{~Tb}$ & $66 \mathrm{Dy}$ & $67 \mathrm{Ho}$ & $68 \mathrm{Er}$ & $69 \mathrm{Tm}$ & $70 \mathrm{Yb}$ & 71 Lu \\
\hline & & & 0,48 & 0,52 & 0,55 & 0,56 & $\mathrm{n} / \mathrm{a}$ & 0,62 & 0,56 & 0,61 & 0,64 & 0,68 & 0,71 & 1,21 & 0,93 & 0,5 & 1,02 \\
\hline & & Actinides & $89 \mathrm{Ac}$ & $90 \mathrm{Th}$ & $91 \mathrm{~Pa}$ & $92 \mathrm{U}$ & $93 \mathrm{~Np}$ & $94 \mathrm{Pu}$ & $95 \mathrm{Am}$ & $96 \mathrm{Cm}$ & $97 \mathrm{Bk}$ & $98 \mathrm{cf}$ & $99 \mathrm{Es}$ & $100 \mathrm{Fm}$ & $101 \mathrm{Md}$ & 102 No & $103 \mathrm{Lr}$ \\
\hline & & & $n / a$ & 0,79 & 0,89 & 0,91 & 0,98 & 0,6 & 1,14 & $\mathrm{n} / \mathrm{a}$ & $\mathrm{n} / \mathrm{a}$ & $n / a$ & $n / a$ & $n / a$ & $\mathrm{n} / \mathrm{a}$ & $\mathrm{n} / \mathrm{a}$ & $\mathrm{n} / \mathrm{a}$ \\
\hline
\end{tabular}

Figure 2. The periodic table of elements with inclusion of value of the quantum concentration for the liquid phase of elements. 


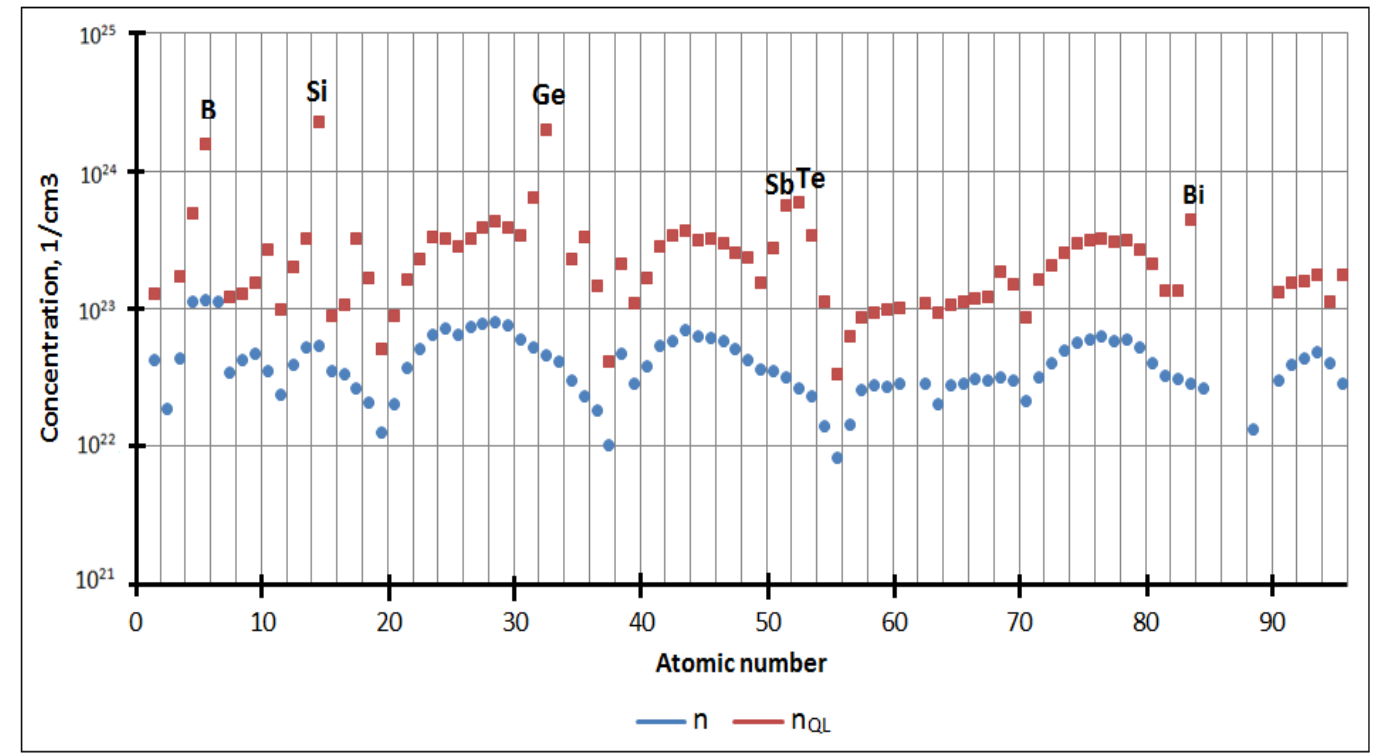

Figure 3. Comparison of quantum concentration of the liquid phase of elements (red) with concentration of atoms (blue). 


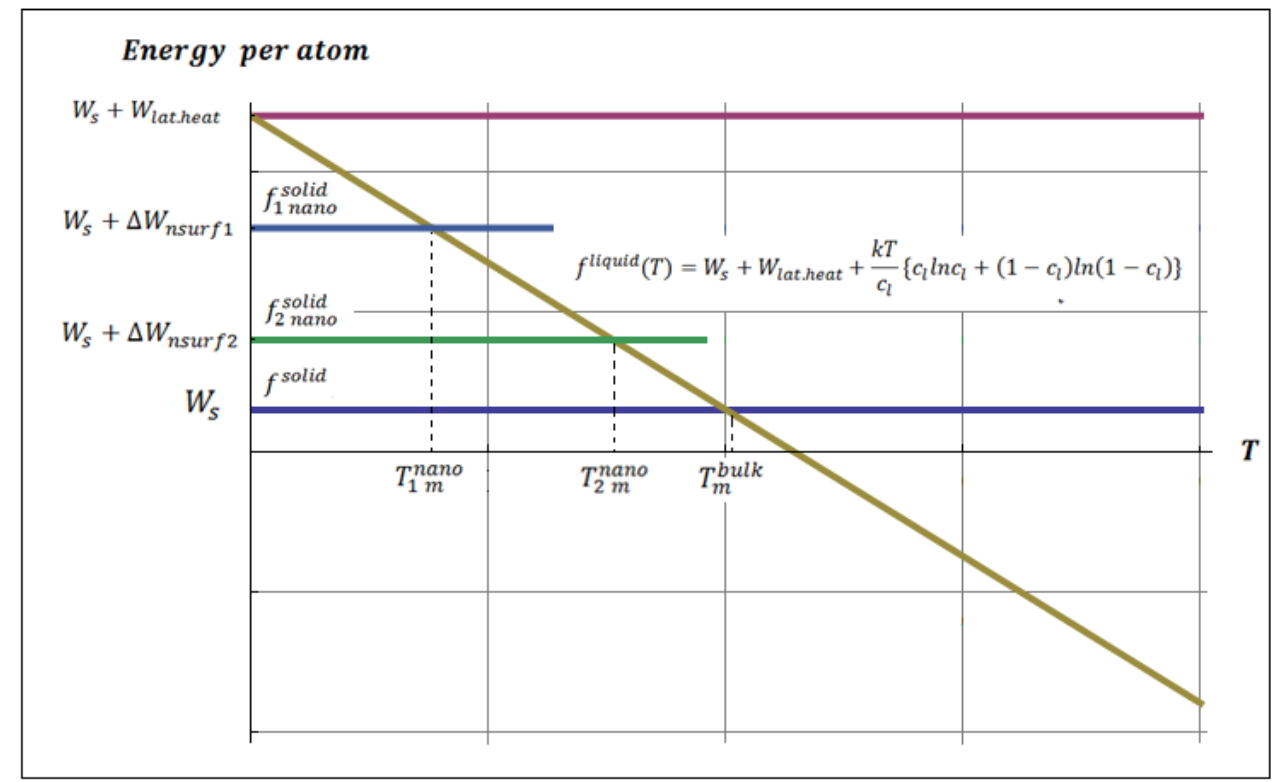

Figure 4. Diagram of the thermodynamic model for a solid-liquid phase transition for nanoparticles. 

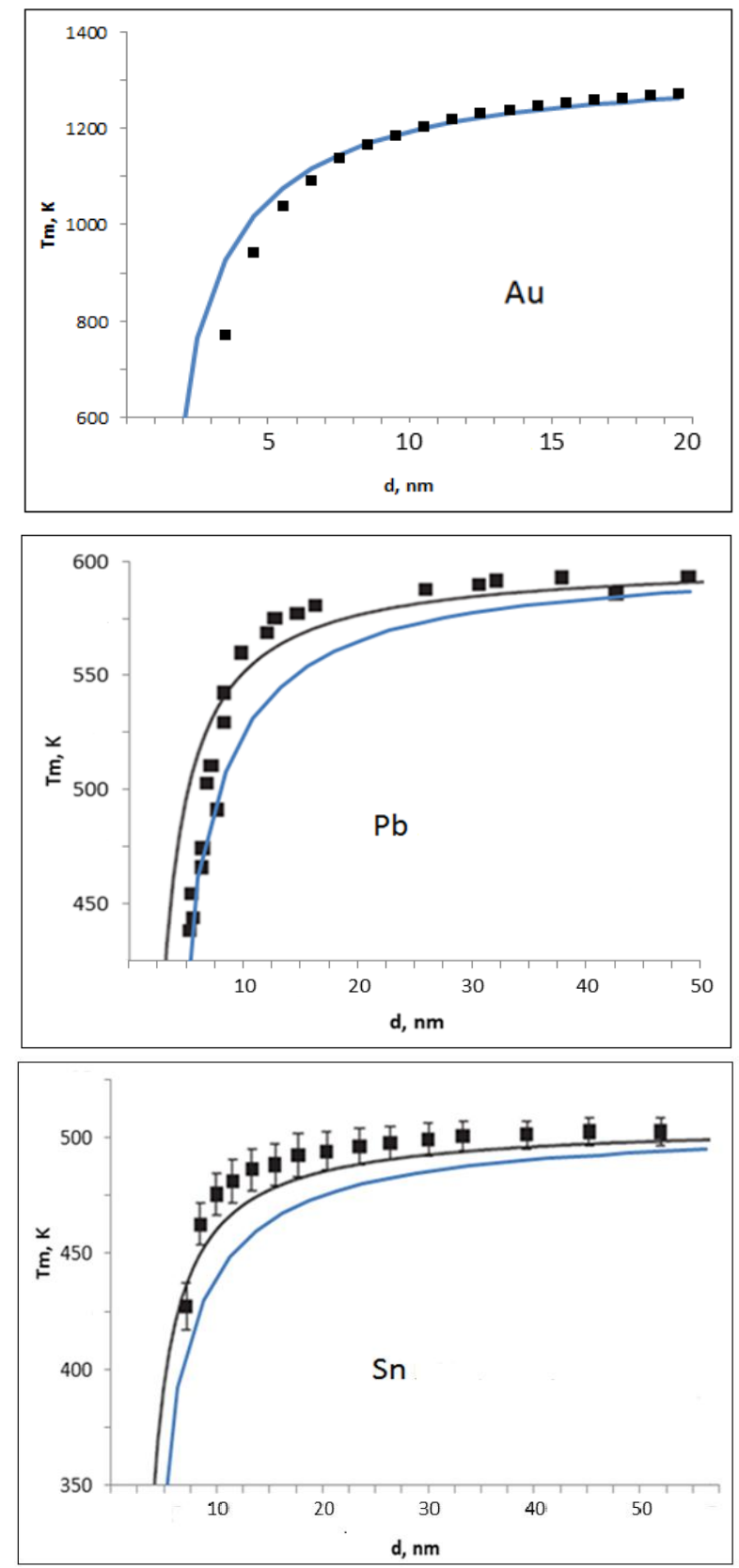

Figure 5. Melting temperature of $\mathrm{Au}, \mathrm{Pb}$ and $\mathrm{Sn}$ nanoparticles as a function of particle diameter. Blue line is calculation on the basis of quantum concentration for liquids. Black line is W.H. Qi model [14]. Symbols are experimental data from Refs. [16-18]. 
GRAPHICAL ABSTRACT

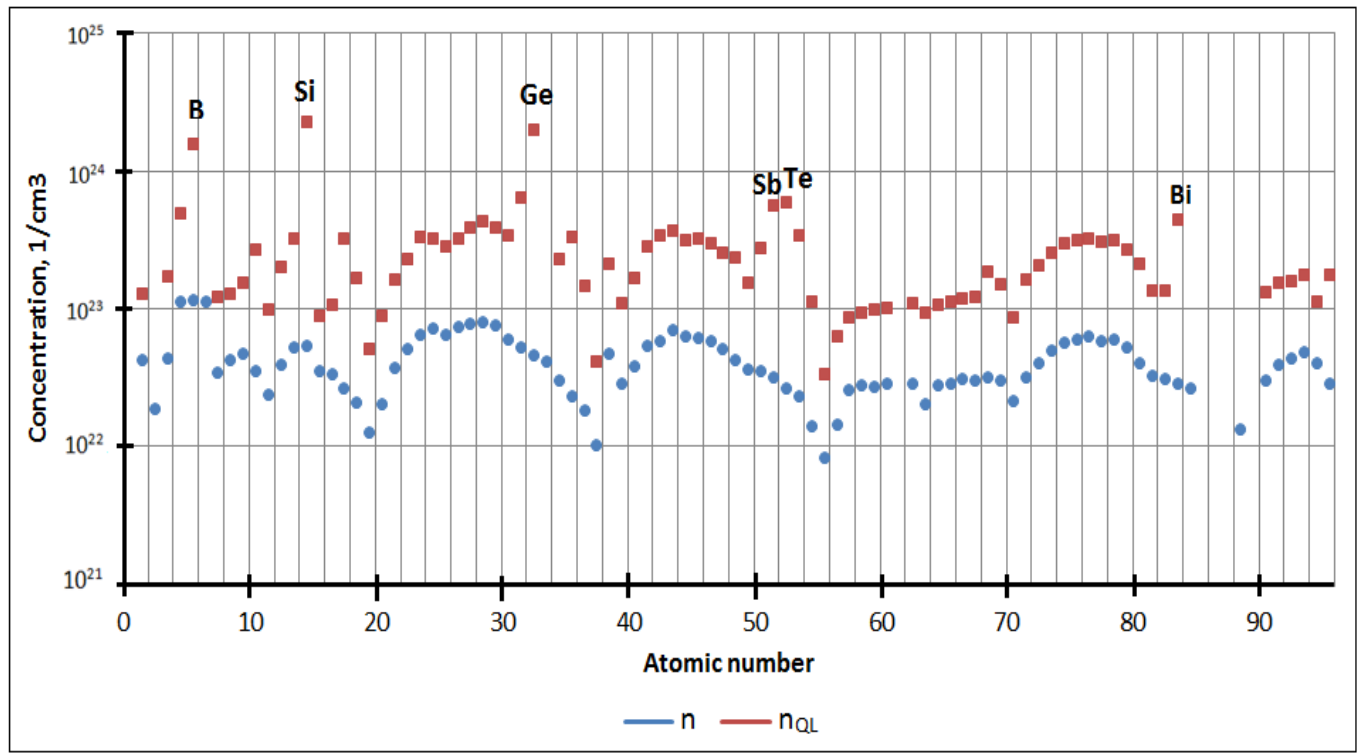

